\title{
Glioblastoma with Extracranial Multiple Metastasis
}

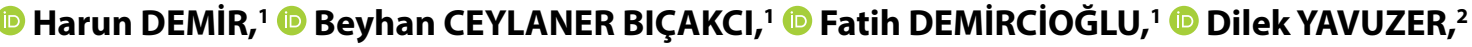 \\ D Gökhan YAPRAK,' (1) Evrim METCALFE ${ }^{3}$
}

\begin{abstract}
'Department of Radiation Oncology, Kartal Dr. Lütfi Kırdar City Hospital, Istanbul-Turkey
${ }^{2}$ Department of Pathology, Sancaktepe Martyr Prof. Dr. IIlhan Varank Training and Research Hospital, Istanbul-Turkey

${ }^{3}$ Department of Radiation Oncology, Biruni University Faculty of Medicine, Istanbul-Turkey
\end{abstract}

Glioblastoma (GB) is the most common malignant central nervous system tumor in adults, accounting for $47.7 \%$ of all primary malignant brain tumors.[1] The main treatment is surgical resection and gross total resection improves overall survival. Temozolomide (TMZ) and adjuvant chemotherapy along with postoperative radiotherapy have also become standardized for patients with GB.[2] The prognosis is very poor and most patients die within 1 year of diagnosis.

GBs usually show local growth and infiltration. Despite the aggressive characteristics of these tumors, extracranial metastases are extremely rare.[3] Incidence is reported to be approximately $0.4-2.0 \%$.[4]

In this study, a case of GB with extensive extracranial metastasis is presented in the light of current literature.

\section{Case 1}

A 23-year-old female patient was admitted to the neurology clinic with the complaint of headache that had lasted for a month, increased in the past week and new onset of vomiting. The patient did not have any additional disease and Karnofsky performance score was $>90 \%$. Magnetic resonance imaging detected a contrast-enhanced mass $(60 \times 54 \times 25 \mathrm{~mm})$ and an area of peripheral edema in the right parietal lobe. The patient was referred to the neurosurgery clinic and underwent total mass resection after craniotomy. Post-operative pathological examination revealed a tumor consisting of atypical cells of glial character with increased mitotic activity and diffusely infiltrating the brain parenchyma. The examination also revealed areas of microvascular proliferation and necrosis. The tumor consisted of fusiform cells forming intersecting bundles with a sarcomatous appearance in some areas. Immunohistochemically, staining with glial fibrillary acidic protein (GFAP) was observed in some of the cells, while staining with isocitrate dehydrogenase 1 was not observed. There was no loss of ATRX expression in tumor cells. The Ki67 proliferative index of the tumor was calculated as $25 \%$. In addition, a significant increase in reticulogenesis was observed in areas that were not stained with reticulin staining and GFAP. With these findings, the tumor was diagnosed as "GB with Gliosarcomatous Component, WHO Grade IV." The patient received concurrent chemoradiotherapy followed by adjuvant TMZ chemotherapy. There was no sign of recurrence in the first 1-year follow-up of the patient. The patient, who did not continue the controls for the following 3 years, applied with the complaint of persistent cough for 3 months. The patient was examined and PET CT revealed multiple mass lesions in the bilateral lung parenchyma and hilum, mediastinal, right axillary, left external iliac and left femoral multiple lymphadenopathies, and central necrotic hypermetabolic lesions in the right arm lateral and left thigh medial muscle. There was no evidence of recurrence in brain MR imaging. The pathology result of the right axillary lymph node excision was reported as GB metastasis (GFAP: Strong positive and Ki67 index: 25\%). The patient received six cycles of bevacizumab and irinotecan chemotherapy in the medical oncology department. PET CT seen for evaluation after chemotherapy was reported as progression. The patient complained of the left thigh pain and limitation of motion, and the left thigh mass biopsy was reported as GB metastasis. The patient received 

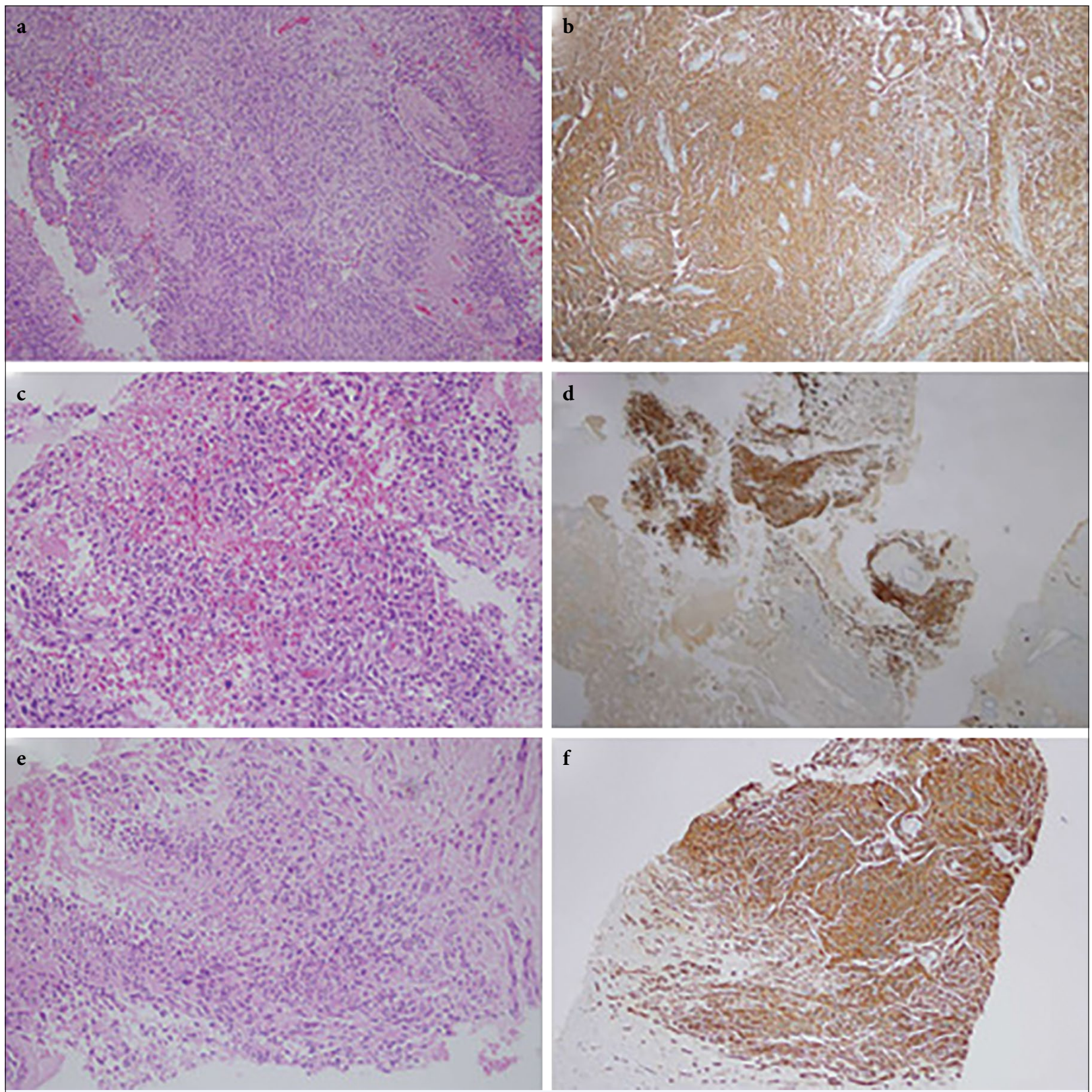

Fig. 1. Histopathology of primary tumor and metastases: $(a, b)$ Primary tumor; pseudopalysed areas of necrosis (H\&E, $\times 40)$ and immunohistochemistry (GFAP, $\times 40)$, (c, d) Right axillary lymph node ; tumor consisting of cells with morphology similar to the tumor in the brain $(\mathrm{H} \& \mathrm{E}, \times 100)$ and immunohistochemistry $(\mathrm{GFAP}, \times 40)$, (e, f) Left thigh; tumor consisting of cells with morphology similar to the tumor in the brain (H\&E, x100) and immunohistochemistry (GFAP, $\times 100) \mathrm{HE}$, hematoxylin and eosin; GFAP, glial fibrillary acidic protein.

GFAP: Glial fibrillary acidic protein.

palliative radiotherapy for the related lesion and the complaint of pain decreased significantly. The patient who received the second series of chemotherapy died while his treatment was continuing, and survival after detection of extensive stage disease was calculated as approximately 16 months (Fig.1).

\section{Discussion}

Extracranial metastases are very rare in GB patients and their mechanisms have not been fully elucidated. Its low incidence is attributed to reasons such as short survival time, presence of blood-brain barrier, and lack 
of lymphatic drainage system in the brain parenchyma. [5] The fact that this case has a relatively long survival time compared to patients with classical GBM supports this hypothesis. The most common sites of extracranial metastases are the lungs, pleura, lymph nodes, and bones.[6] This case also progressed with lung, lymph nodes, and muscle involvement. Pietschmann et al.[7] reported in their meta-analysis that younger patients were more prone to develop extracranial metastases and explained this with longer survival times. The fact that the case was 23 years old supports this hypothesis.

Gliosarcoma (GS) is considered a rare variant of GB (2\%) containing both a gliomatous and a sarcomatous component.[8] Compared to GBs, GSs have a greater propensity for extracranial metastases and are reported in approximately $11 \%$.[9] Smith et al.[10] reported in their case series that the sarcomatous component has a tendency to spread hematogenously and that the metastatic potential of GSs can be attributed to the sarcomatous component. In this context, the primary histopathology of this case was GB with a gliosarcomatous component, indicating a higher risk for the development of extracranial metastases. For this reason, especially patients with GS should be examined for extracranial metastases during the diagnosis and follow-up period.

Lun et al.[11] reported that the survival of GB patients with extracranial metastases who underwent intensive treatment schemes including surgery, radiotherapy, and chemotherapy is longer than those who received less treatment. However, there is no treatment recommendation with proven survival benefit, and a balance must be struck between survival, quality of life, and toxicity when planning treatment.[7] This case also received intensive treatment including chemotherapy and radiotherapy because of good performance. However, the desired response could not be obtained due to disease progression.

\section{Conclusion}

Oncology professionals should always keep in mind the risk of distant metastasis in patients with GB and especially GS, even if extracranial metastases are quite rare.

\section{References}

1. Ostrom QT, Gittleman H, Truitt G, Boscia A, Kruchko C, Barnholtz-Sloan JS. CBTRUS statistical report: Pri- mary brain and other central nervous system tumors diagnosed in the United States in 2011-2015. Neuro Oncol 2018;20 Suppl 4:iv1-86.

2. Reni M, Mazza E, Zanon S, Gatta G, Vecht CJ. Central nervous system gliomas. Crit Rev Oncol Hematol 2017;113:213-34.

3. Zhen L, Yufeng C, Zhenyu S, Lei X. Multiple extracranial metastases from secondary glioblastoma multiforme: A case report and review of the literature. J Neurooncol 2010;97(3):451-7.

4. Hamilton JD, Rapp M, Schneiderhan T, Sabel M, Hayman A, Scherer A, et al. Glioblastoma multiforme metastasis outside the CNS: Three case reports and possible mechanisms of escape. J Clin Oncol 2014;32(22):e80-4.

5. Xu M, Wang Y, Xu J, Yao Y, Yu WX, Zhong P. Extensive therapies for extraneural metastases from glioblastoma, as confirmed with the OncoScan assay. World Neurosurg 2016;90:698.e7-11.

6. Piccirilli M, Brunetto GM, Rocchi G, Giangaspero F, Salvati M. Extra central nervous system metastases from cerebral glioblastoma multiforme in elderly patients. Clinico-pathological remarks on our series of seven cases and critical review of the literature. Tumori 2008;94(1):40-51.

7. Pietschmann S, von Bueren AO, Henke G, Kerber MJ, Kortmann RD, Müller K. An individual patient data meta-analysis on characteristics, treatments and outcomes of the glioblastoma/gliosarcoma patients with central nervous system metastases reported in literature until 2013. J Neurooncol 2014;120(3):451-7.

8. Ben Nsir A, Thai QA, Kassar AZ, Ben Said I, Jemel H. Primary cerebellar gliosarcoma with extracranial metastases: An orphan differential diagnosis. World Neurosurg 2015;84(6):2076.e13-7.

9. Beaumont TL, Kupsky WJ, Barger GR, Sloan AE. Gliosarcoma with multiple extracranial metastases: Case report and review of the literature. J Neurooncol 2007;83(1):39-46.

10. Smith DR, Hardman JM, Earle KM. Contiguous glioblastoma multiforme and fibrosarcoma with extracranial metastasis. Cancer 1969;24(2):270-6.

11. Lun M, Lok E, Gautam S, Wu E, Wong ET. The natural history of extracranial metastasis from glioblastoma multiforme. J Neurooncol 2011;105(2):261-73. 